\title{
Implementation of a Distributed Data Mining System
}

\author{
Ju Cho ${ }^{1}$, Sung Baik ${ }^{1,}$, and Jerzy Bala ${ }^{2}$ \\ ${ }^{1}$ Sejong University, Seoul 143-747, Korea \\ \{jscho, sbaik\} asejong.ac.kr \\ ${ }^{2}$ Datamat Systems Research, Inc. \\ 1600 International Drive, McLean, VA 22102, USA \\ jbala@dsri.com
}

\begin{abstract}
This paper describes the implementation of a distributed data mining system. The system consists of a web server, a pre-processor for data preparation, a mediator, and agents. A distributed learning algorithm of a decision tree in an agent-mediator communication mechanism is the most important and difficult to achieve the distributed data mining in this system, in the view of implementation. The algorithm has successfully been implemented with several techniques. Its implementation is presented in a UML (Unified Modeling Language) sequence diagram.
\end{abstract}

\section{Introduction}

In this paper, we consider a distributed data mining (DDM) approach [1], in which the modified decision tree algorithm on an agent based framework can deal with heterogeneous data sets in the distributed environment $[2,3]$. The data mining based on the algorithm takes full advantage of all the available data through a mechanism for integrating data from a wide variety of data sources and is able to handle data characterized by geographic (or logical) distribution, complexity and multi-feature representations, and the vertical partitioning/distribution of feature sets.

The paper describes the implementation of an agent based distributed data mining system which consists of a web server, a pre-processor for data preparation, a mediator, and agents. The web server supports users with a web-based interface through which they can access databases located at different sites and manipulate data mining facilities. The pre-processor prepares data sets for data mining by dealing with databases in a distributed way. The implementation of data preparation for DDM is presented in section 2. The mediator coordinates the communication between several agents with security concerns such as authentication. Each agent is located at each heterogeneous data site to achieve coordinated learning through the cooperation of local learning and communication with the other agents. The implementation of the communication, between the mediator and agents for DDM, and the distributed learning algorithm of a decision tree in the communication mechanism is presented in section 3 .

"Author for Correspondence. 


\section{Data Preparation for Distributed Data Mining}

Data preparation is a preliminary and necessary phase for data mining. In DDM, data sets located in different distributed sites should be processed and converted to appropriate data forms in advance, so that data mining can be performed in a distributed fashion. Fig. 1 shows a UML sequence diagram for data preparation. In our work, two kinds of files are generated from the database for DDM; 1) a control file (e.g. data.ctr) and 2) a learning file (e.g. data.lea). The steps of data preparation are as follows:

1. The selected database schema information and the meta-data from all of different distributed sites are exchanged via communication between the mediator and agents. Each agent deals with each database at its own location.

2. According to the exchanged information, control files are generated at the sites participating for data analysis. Each control file has all schema information obtained from all databases located at different sites.

3. The values of the class field selected by a user are transferred to other sites from the site which the class field belongs to. The class information is found in all example control files presented in Fig. 2.

4. A primary key for associating the data across different sites is selected. The only co-existing values of the primary key field in all databases are selected. And then their associated tuples are selected to build a learning file in each site.

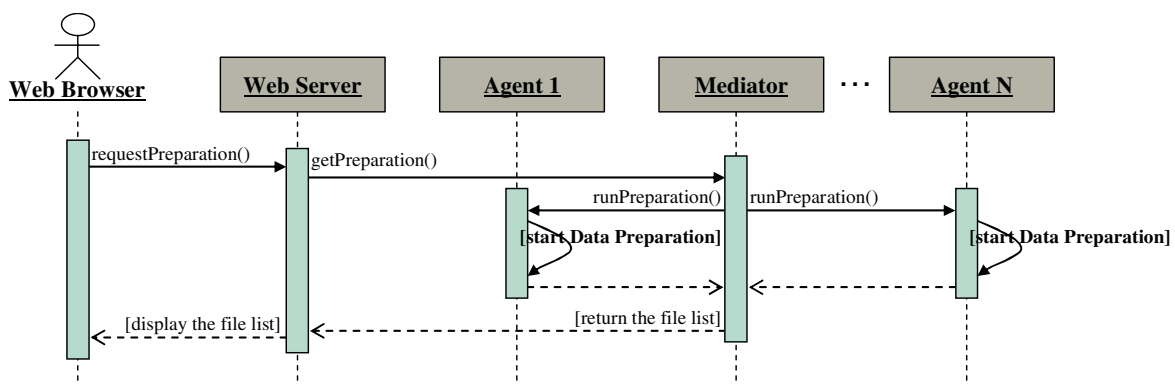

Fig. 1. UML Sequence Diagram for Data Preparation for DDM

\section{Distributed Data Mining}

A data mining engine was implemented in $\mathrm{C}$ in a previous work [4]. The communication interface within the agent was implemented in Java. The communication of the mediator and the agents was implemented in Java on RMI (Remote Method Interface). The data mining engine implemented in $\mathrm{C}$ is interfaced with the communication interface implemented in Java by JNI (Java Native Interface). The interfaces of core remote methods for DDM are presented in Fig. 3. 
Before Data Preparation

\begin{tabular}{|c|c|c|c|}
\hline pkey & $\mathbf{X 1}$ & $\mathbf{X 2}$ & $\mathbf{X 3}$ \\
\hline 001 & 1.3 & 10 & 23 \\
\hline 002 & 2.1 & 15 & 91 \\
\hline 004 & 0.5 & 15 & 11 \\
\hline 005 & 1.9 & 10 & 17 \\
\hline 007 & 1.2 & 10 & 21 \\
\hline
\end{tabular}

DB 1

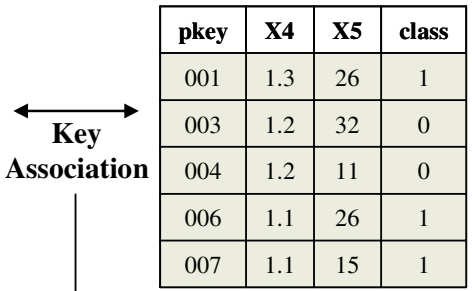

DB 2

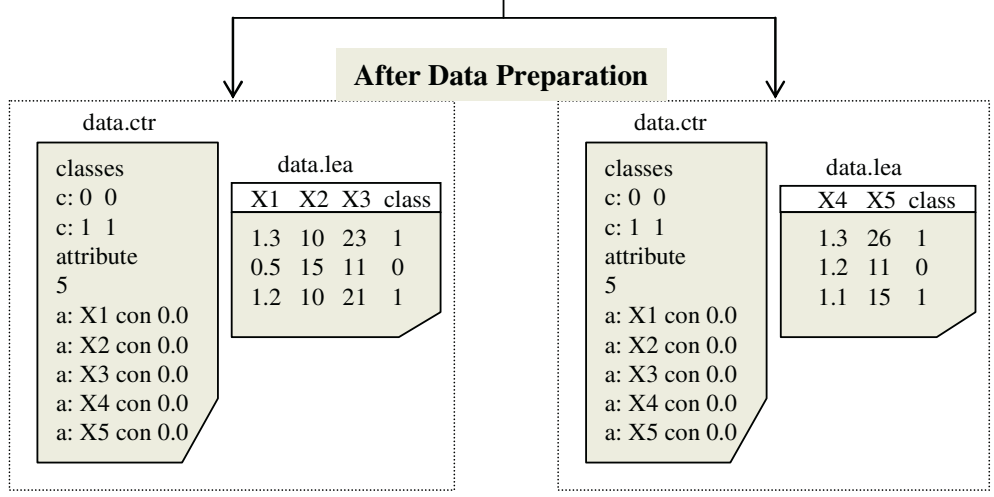

Fig. 2. Examples of Data Sets before/after Data Preparation

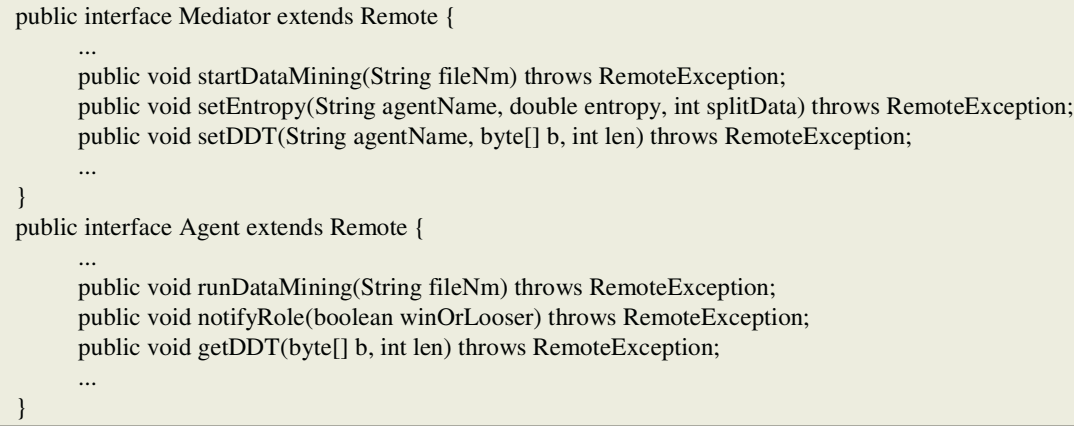

Fig. 3. Interface Methods for the Communication between Mediator and Agent

Fig. 4 presents a UML sequence diagram for DDM. The distributed learning algorithm of a decision tree in an agent-mediator communication mechanism is as follows:

1. runDataMining() : Start the local data mining processes associated with local agents.

2. [find the attribute] : Find the attribute and its associated value that can best split the data into the various training classes during local mining. 
3. setEntropy (): Send the best local attribute and its associated value to the mediator.

4. [select the best attribute] : Select the best attribute from the best local attributes of all the agents.

5. notifyRole() : Notify each agent of its role for the next action (splitting or waiting).

6. [split the data] : Split the data, according to the best global attribute and its associated split value, in the formation of two separate clusters of data in the selected agent.

7. setDDT ()$\&$ getDDT () : Distribute the structural information in each cluster and the best attribute to the other agents through the mediator.

8. [construct the partial decision tree] : Construct the partial decision trees according to the structural information in other agents.

9. [generate rules] : Generate decision rules at each agent and notify the mediator for termination if there is no more splitting. Otherwise, go to step 2.

10. [notify the end of DDM] : Terminate.

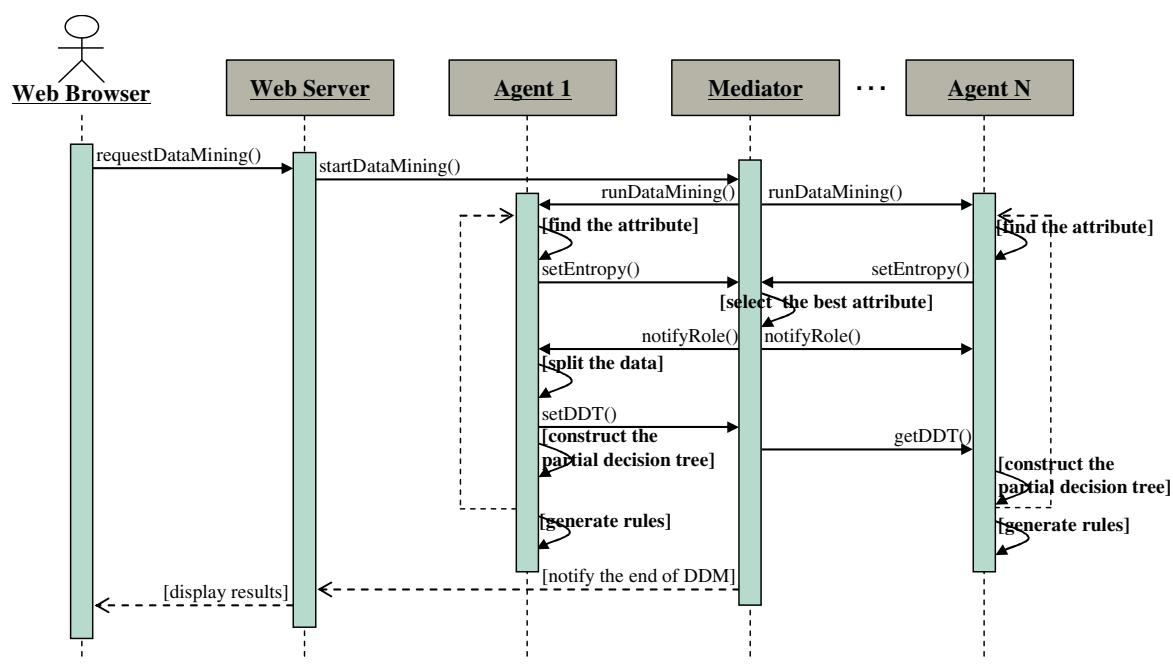

Fig. 4. UML Sequence Diagram for Distributed Data Mining

\section{References}

1. S. W. Baik, J. Bala and J. S. Cho: Agent Based Distributed Data Mining, Lecture Notes in Computer Science, Vol. 3320, pp.42-45, 2004

2. D. Caragea, A. Silvescu and V. Honavar: Decision Tree Induction from Distributed, Heterogeneous, Autonomous Data Sources, Proceedings of the Conference on Intelligent Systems Design and Applications (ISDA 03), 2003

3. C. Giannella, K. Liu, T. Olsen and H. Kargupta: Communication Efficient Construction of Decision Trees Over Heterogeneously Distributed Data, Proceedings of the Fourth IEEE International Conference on Data Mining (ICDM 04), pp.67-74, 2004

4. J. Bala, S. W. Baik, S. Gutta and P. Pachowicz: InferView: An Integrated System for Knowledge Acquisition and Visualization, Proceedings of AFCEA Federal Data Mining Symposium, 1999 